\title{
ON THE SPECTRAL AND MODULATIONAL STABILITY OF PERIODIC WAVETRAINS FOR NONLINEAR KLEIN-GORDON EQUATIONS*
}

\author{
CHRISTOPHER K. R. T. JONES, ROBERT MARANGELL, PETER D. MILLER, \\ AND RAMÓN G. PLAZA
}

\begin{abstract}
In this contribution, we summarize recent results $[8,9]$ on the stability analysis of periodic wavetrains for the sine-Gordon and general nonlinear Klein-Gordon equations. Stability is considered both from the point of view of spectral analysis of the linearized problem and from the point of view of the formal modulation theory of Whitham [12]. The connection between these two approaches is made through a modulational instability index [9], which arises from a detailed analysis of the Floquet spectrum of the linearized perturbation equation around the wave near the origin. We analyze waves of both subluminal and superluminal propagation velocities, as well as waves of both librational and rotational types. Our general results imply in particular that for the sine-Gordon case only subluminal rotational waves are spectrally stable. Our proof of this fact corrects a frequently cited one given by Scott [11].
\end{abstract}

\section{INTRODUCTION}

1.1. Periodic traveling waves. In this paper we consider nonlinear Klein-Gordon equations of the form

$$
u_{t t}-u_{x x}+V^{\prime}(u)=0
$$

where $u$ is a scalar function of $(x, t) \in \mathbb{R} \times[0,+\infty)$ and the potential $V$ is a real periodic function. Such potentials generalize the case $V(u)=-\cos (u)$, for which equation (1.1) becomes the well-known sine-Gordon equation [10] in laboratory coordinates,

$$
u_{t t}-u_{x x}+\sin (u)=0 .
$$

To facilitate the exposition we shall assume that $V: \mathbb{R} \rightarrow \mathbb{R}$ is a periodic function of class $C^{2}$ and that $V$ has exactly two non-degenerate critical points per period. Moreover, after an appropriate scaling we can also assume, without loss of generality, that the potential $V$ has fundamental period $2 \pi$, and $\min _{u \in \mathbb{R}} V(u)=-1$, while $\max _{u \in \mathbb{R}} V(u)=1$. (The sine-Gordon potential clearly satisfies these hypotheses.) While the above assumptions on $V$ allow for an easier exposition, many of our results also hold for more general periodic $V$, and even the assumption of periodicity of $V$ can be dropped in some cases (see [9] for further information). Equation (1.1) has traveling wave solutions of the form

$$
u(x, t)=f(z), \quad z:=x-c t
$$

where $c \in \mathbb{R}$ is the wave speed. In what follows we shall assume that $c \neq \pm 1$. Substituting into (1.1) we readily see that the profile function $f: \mathbb{R} \rightarrow \mathbb{R}$ satisfies the nonlinear ordinary differential equation

$$
\left(c^{2}-1\right) f_{z z}+V^{\prime}(f)=0 .
$$

* Prepared for the Proceedings of the XV International Conference on Hyperbolic Problems: Theory, Numerics, Applications (HYP2014). July 28 - August 1, 2014. IMPA, Rio de Janeiro, Brazil. 
Equation (1.4) can be integrated once to obtain

$$
\frac{1}{2}\left(c^{2}-1\right) f_{z}^{2}=E-V(f),
$$

where $E$ is an integration constant with the interpretation of total (kinetic plus potential) energy. Periodic traveling waves of (1.1) can be classified according to the values of $E$ and $c$. The first dichotomy concerns the wave speed: if $c^{2}<1$ then $f$ is called a subluminal periodic traveling wave; if $c^{2}>1$ then $f$ is called a superluminal periodic traveling wave. The second dichotomy involves parameter values of the energy $E$. We call solutions to the pendulum equation (1.5) whose orbits in the phase plane lie outside the separatrix rotational waves. Solutions whose orbits in the phase plane lie inside the separatrix are called librational waves. It is easy to see that librational waves correspond to energies in the range $|E|<1$, for which $f(z+T)=f(z)$ for all $z \in \mathbb{R}$ and some fundamental period $T>0$. On the other hand, rotational waves correspond to energies with either $E>1$ (in the superluminal case), or $E<-1$ (in the subluminal case). Rotational waves satisfy $f(z+T)=f(z) \pm 2 \pi$ for all $z \in \mathbb{R}$. These waves are also called kink trains (or antikink trains, depending on the sign of $f_{z}$ ) in the literature. See Figure 1.
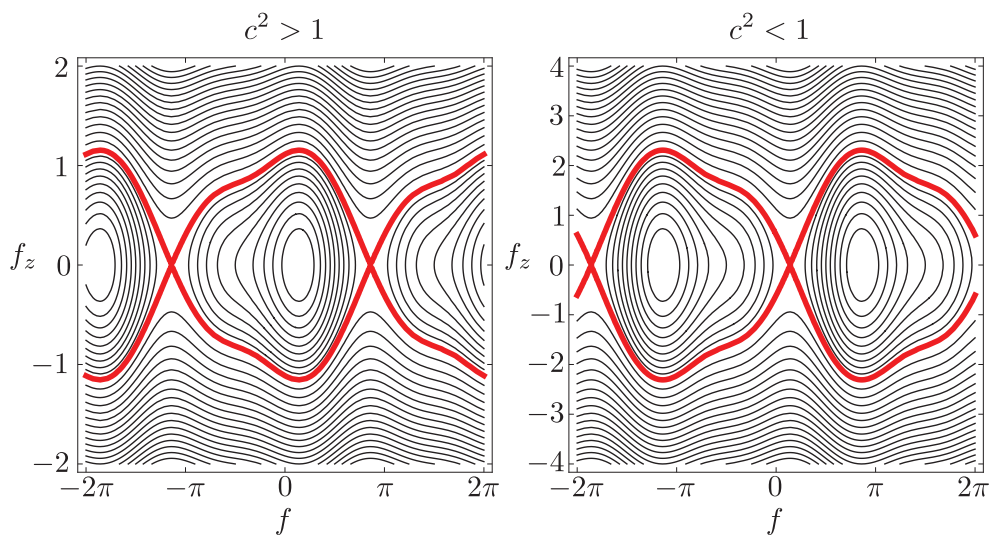

Figure 1. Phase portraits of equation (1.4) for $c=2$ (left) and $c=1 / 2$ (right), where the potential is $V(u)=-0.861\left[\cos (u)+\frac{1}{3} \sin (2 u)\right]$, which satisfies the basic assumptions under consideration. The separatrices are the thicker red curves. (Color online.)

1.2. Monotonicity of the period map. Some of our results require that a non-degeneracy condition is satisfied, namely, that the energy is not a critical point of the period. Although this condition is always satisfied for rotational waves, further assumptions on the potential need to be verified in the librational case. A sufficient condition was introduced by Chicone [3]. The precise statement for the waves under consideration goes as follows (see Propositions 2.10 and 2.11 in [9] for the proof).

Proposition $1.1([9])$. For rotational waves, the period $T$ is a strictly monotone function of the energy, and $\left(c^{2}-1\right) T_{E}<0$. If also $V$ is of class $C^{3}$ and the functions

$$
N^{ \pm}: \mathbb{R} \rightarrow \mathbb{R}, \quad N^{ \pm}(f):=6[V(f) \pm 1] V^{\prime \prime}(f)^{2}-3 V^{\prime}(f)^{2} V^{\prime \prime}(f)-2[V(f) \pm 1] V^{\prime}(f) V^{\prime \prime \prime}(f),
$$


are both not identically zero and semidefinite (Chicone's criterion [3]), then $T_{E} \neq 0$ holds for all librational waves, and the sign of $T_{E}$ coincides with that of $N^{+}$(resp., $N^{-}$) for superluminal (resp., subluminal) waves.

Finally, it is to be observed that with a particular choice of origin for $z$, the periodic wavetrain $f=f(z ; E, c)$ is uniquely determined and for each $z$ is a $C^{2}$ function of $(E, c)$ in a four-component, non-connected, open set (see Lemma 2.8 in [9] for further details).

\section{The stability problem. Floquet spectrum}

2.1. Perturbation equations. Let us now consider a perturbation of the periodic traveling wave $f=f(z)$. Substituting $u=f+v$ into the Klein-Gordon equation (1.1) written in the galilean frame associated with the independent variables $(z=x-c t, t)$ and using the equation (1.4) satisfied by $f$, one finds that the perturbation $v$ necessarily satisfies the nonlinear equation

$$
v_{t t}-2 c v_{z t}+\left(c^{2}-1\right) v_{z z}+V^{\prime}(f(z)+v)-V^{\prime}(f(z))=0 .
$$

Specializing to perturbations of the form $v(z, t)=w(z) e^{\lambda t}$, where $\lambda \in \mathbb{C}$, and after linearizing around $v=0$ we obtain the linear ODE

$$
\left(c^{2}-1\right) w_{z z}-2 c \lambda w_{z}+\left(\lambda^{2}+V^{\prime \prime}(f(z))\right) w=0,
$$

in which the complex growth rate $\lambda$ appears as a (spectral) parameter. Roughly speaking, a necessary condition for the stability of $f$ is that there are no points of spectrum with $\operatorname{Re} \lambda>0$ (which would imply the existence of a solution $w$ of (2.2) that lies in a Banach space $X$ as a function of $z$, and grows exponentially in time). Following Alexander, Gardner and Jones [1], the spectral problem (2.2) with $w \in X$ can be equivalently regarded as a first order system of the form

$$
\mathbf{w}_{z}=\mathbf{A}(z, \lambda) \mathbf{w}
$$

where $\mathbf{w}:=\left(w, w_{z}\right)^{\top} \in Y(Y$ is a Banach space related to $X)$, and

$$
\mathbf{A}(z, \lambda):=\left(\begin{array}{cc}
0 & 1 \\
-\frac{\left(\lambda^{2}+V^{\prime \prime}(f(z))\right)}{c^{2}-1} & \frac{2 c \lambda}{c^{2}-1}
\end{array}\right) .
$$

Note that the coefficient matrix $\mathbf{A}$ is periodic in $z$ with period $T$. Clearly, the definition of the spectrum depends upon the choice of the space $X$. If $X=L^{2}(\mathbb{R} ; \mathbb{C})$ then the analysis corresponds to stability under localized perturbations. It is well-known [4] that the $L^{2}(\mathbb{R})$ spectrum of a differential operator with periodic coefficients is purely "continuous" (there are not isolated eigenvalues).

2.2. Floquet characterization of the spectrum. The periodic Evans function. Let $\mathbf{F}(z, \lambda)$ denote the $2 \times 2$ identity-normalized fundamental solution matrix for the differential equation (2.3), i.e., the unique solution of

$$
\mathbf{F}_{z}(z, \lambda)=\mathbf{A}(z, \lambda) \mathbf{F}(z, \lambda), \quad \text { with initial condition } \mathbf{F}(0, \lambda)=\mathbb{I}, \forall \lambda \in \mathbb{C} .
$$

The $T$-periodicity in $z$ of the coefficient matrix $\mathbf{A}$ then implies that

$$
\mathbf{F}(z+T, \lambda)=\mathbf{F}(z, \lambda) \mathbf{M}(\lambda), \quad \forall z \in \mathbb{R}, \quad \text { where } \quad \mathbf{M}(\lambda):=\mathbf{F}(T, \lambda) .
$$

The matrix $\mathbf{M}(\lambda)$ is called the monodromy matrix for the first-order system (2.3), and its elements are entire functions of $\lambda \in \mathbb{C}$ (due to analyticity of $\mathbf{A}$ in $\lambda \in \mathbb{C}$ and uniform convergence of Picard iterates for bounded $z$ ). Let $\mu(\lambda)$ denote an eigenvalue of $\mathbf{M}(\lambda)$, and 
let $\mathbf{w}^{0}(\lambda) \in \mathbb{C}^{2}$ denote a corresponding (nonzero) eigenvector. Then $\mathbf{w}(z, \lambda):=\mathbf{F}(z, \lambda) \mathbf{w}^{0}(\lambda)$ is a nontrivial solution of the first-order system (2.3) that satisfies

$$
\begin{aligned}
\mathbf{w}(z+T, \lambda) & =\mathbf{F}(z+T, \lambda) \mathbf{w}^{0}(\lambda)=\mathbf{F}(z, \lambda) \mathbf{M}(\lambda) \mathbf{w}^{0}(\lambda) \quad(\text { by }(2.6)) \\
& =\mu(\lambda) \mathbf{F}(z, \lambda) \mathbf{w}^{0}(\lambda)=\mu(\lambda) \mathbf{w}(z, \lambda), \quad \forall z \in \mathbb{R} .
\end{aligned}
$$

Such solutions are called Floquet solutions, and the eigenvalue $\mu(\lambda)$ of the monodromy matrix $\mathbf{M}(\lambda)$ is called a Floquet multiplier. If $R(\lambda)$ denotes any number (modulo $2 \pi i$ ) for which $e^{R(\lambda)}=\mu(\lambda)$, then $e^{-R(\lambda) z / T} \mathbf{w}(z, \lambda)$ is a $T$-periodic function of $z$, or, equivalently (Bloch's Theorem $) \mathbf{w}(z, \lambda)$ can be written in the form

$$
\mathbf{w}(z, \lambda)=e^{R(\lambda) z / T} \mathbf{z}(z, \lambda), \quad \text { where } \mathbf{z}(z+T, \lambda)=\mathbf{z}(z, \lambda), \forall z \in \mathbb{R} .
$$

The quantity $R(\lambda)$ is sometimes called a Floquet exponent. A further consequence of Floquet theory is that if the first-order system (2.3) has a nontrivial solution in $L^{\infty}\left(\mathbb{R}, \mathbb{C}^{2}\right)$, it is necessarily a linear superposition of Floquet solutions corresponding to Floquet multipliers $\mu(\lambda)$ with $|\mu(\lambda)|=1$. Thus, one can parametrize the spectrum according to values of $\mu=e^{i \theta} \in S^{1}$, or equivalently $\theta \in \mathbb{R}(\bmod 2 \pi)$, by introducing the set $\sigma_{\theta}$ of complex numbers $\lambda$ for which there exists a nontrivial solution of the boundary-value problem (2.2) with the condition

$$
\left(\begin{array}{c}
w(T) \\
w_{z}(T)
\end{array}\right)=e^{i \theta}\left(\begin{array}{c}
w(0) \\
w_{z}(0)
\end{array}\right), \quad \theta \in \mathbb{R} .
$$

Obviously the sets $\sigma_{\theta}$ and $\sigma_{\theta+2 \pi n}$ coincide for all $n \in \mathbb{Z}$. The spectrum $\sigma$ may be defined as the union of these partial spectra as follows:

$$
\sigma=\bigcup_{-\pi<\theta \leq \pi} \sigma_{\theta},
$$

and it is characterized in terms of the monodromy matrix through its determinant (see [9], Proposition 3.4):

Proposition 2.1. $\lambda \in \sigma$ if and only if there exists $\mu \in \mathbb{C}$ with $|\mu|=1$ such that

$$
D(\lambda, \mu):=\operatorname{det}(\mathbf{M}(\lambda)-\mu \mathbb{I})=0,
$$

that is, at least one of the Floquet multipliers lies on the unit circle.

Definition 2.2 (periodic Evans function; Gardner [4]). The periodic Evans function is the restriction of $D(\lambda, \mu)$ to the unit circle $S^{1} \subset \mathbb{C}$ in the second argument, which is to be regarded as a unitary parameter in this context. Thus, for each $\theta \in \mathbb{R}(\bmod 2 \pi), D\left(\lambda, e^{i \theta}\right)$ is an entire function of $\lambda \in \mathbb{C}$ whose (isolated) zeros are particular points of the spectrum $\sigma$.

Each set $\sigma_{\theta}$ is characterized as the zero set of the (entire in $\lambda$ ) periodic Evans function $D\left(\lambda, e^{i \theta}\right)$ and hence is purely discrete. The discrete partial spectrum $\sigma_{\theta}$ can therefore be detected for fixed $\theta \in \mathbb{R}$ by standard techniques based on the use of the Argument Principle. However, the study of localized perturbations requires considering all of the partial spectra $\sigma_{\theta}$ at once. The real angle parameter $\theta$ is typically a local coordinate for the spectrum $\sigma$ as a real subvariety of the complex $\lambda$-plane. This explains the intuition that the $L^{2}$ spectrum is purely "continuous", and gives rise to the notion of curves of spectrum (see Proposition 3.7 in $[9])$.

We say that the periodic wave $f$ is spectrally stable if there are no curves of spectrum with $\operatorname{Re} \lambda>0$. Since equation (1.1) is a real Hamiltonian system, this implies that $\sigma$ is symmetric with respect to reflection in the real and imaginary axes, i.e., if $\lambda \in \sigma$, then also 
$\lambda^{*} \in \sigma$ and $-\lambda \in \sigma$ (and hence also $-\lambda^{*} \in \sigma$ ). Thus, spectral stability reduces to the curve $\sigma$ being confined to the imaginary axis.

\section{AnALYSis OF THE MONODROMY MATRIX}

3.1. Series expansions. To study the behavior of the monodromy matrix near the origin we start with the solutions at $\lambda=0$. It can be proved (see Lemma 5.1 in [9]) that the two-dimensional complex vector space of solutions to the first-order system (2.3) at $\lambda=0$ is spanned by

$$
\mathbf{w}^{z}(z):=\left(\begin{array}{c}
f_{z} \\
f_{z z}
\end{array}\right) \text { and } \mathbf{w}^{E}(z):=\left(\begin{array}{c}
f_{E} \\
f_{E z}
\end{array}\right) .
$$

We use this information in a key way to prove the following:

Proposition 3.1 ([9]). The monodromy matrix $\mathbf{M}(0)$ is given by

$$
\mathbf{M}(0)=\left(\begin{array}{cc}
1 & -\left(c^{2}-1\right) T_{E} v_{0}^{2} \\
0 & 1
\end{array}\right) .
$$

Here, $v_{0}^{2}=f_{z}\left(z_{0}\right)^{2}$ where $z_{0}$ is such that $V^{\prime}\left(f\left(z_{0}\right)\right)=0$. In particular, $\mathbf{M}(0)$ is not diagonalizable unless $T_{E}=0$.

The fundamental solution matrix $\mathbf{F}(z, \lambda)$ has a convergent Taylor expansion about every point of the complex $\lambda$-plane. In particular, the series about the origin has the form $\mathbf{F}(z, \lambda)=$ $\sum_{n=0}^{\infty} \lambda^{n} \mathbf{F}_{n}(z), z \in[0, T]$, for some coefficient matrices $\left\{\mathbf{F}_{n}(z)\right\}_{n=0}^{\infty}$, and this series has an infinite radius of convergence. Setting $\lambda=0$ gives $\mathbf{F}_{0}(z)=\mathbf{F}(z, 0)$. By the method of variation of parameters, one arrives at a hierarchy of ODEs for the elements of the expansion, and it is possible to explicitly compute $\mathbf{F}_{1}(z)$ and $\mathbf{F}_{2}(z)$ :

$$
\begin{gathered}
\mathbf{F}_{1}(z)=\frac{c z \mathbf{F}_{0}(z)}{c^{2}-1}+\frac{c\left[\sigma_{-}, \mathbf{F}_{0}(z)\right]}{c^{2}-1}, \quad \sigma_{-}:=\left(\begin{array}{cc}
0 & 0 \\
1 & 0
\end{array}\right) \\
\mathbf{F}_{2}(z)=\frac{1}{2} \frac{c^{2} z^{2} \mathbf{F}_{0}(z)}{\left(c^{2}-1\right)^{2}}+\frac{c^{2} z\left[\sigma_{-}, \mathbf{F}_{0}(z)\right]}{\left(c^{2}-1\right)^{2}}-\frac{c^{2} \sigma_{-} \mathbf{F}_{0}(z) \sigma_{-}}{\left(c^{2}-1\right)^{2}} \\
\quad+\frac{\mathbf{F}_{0}(z)}{\left(c^{2}-1\right)^{2}} \int_{0}^{z} \mathbf{F}_{0}(y)^{-1} \sigma_{-} \mathbf{F}_{0}(y) d y,
\end{gathered}
$$

where $[\mathbf{A}, \mathbf{B}]:=\mathbf{A B}-\mathbf{B A}$ denotes the matrix commutator. Setting $z=T$ in the series formula for $\mathbf{F}(z, \lambda)$ gives the series for the monodromy matrix $\mathbf{M}(\lambda)$, also an entire function of $\lambda: \mathbf{M}(\lambda)=\sum_{n=0}^{\infty} \lambda^{n} \mathbf{M}_{n}$, with $\mathbf{M}_{n}:=\mathbf{F}_{n}(T)$. From (3.3) we then obtain

$$
\begin{aligned}
\mathbf{M}_{1}=q \mathbf{M}(0)+ & \frac{c\left[\sigma_{-}, \mathbf{M}(0)\right]}{c^{2}-1} \\
\mathbf{M}_{2}= & \frac{1}{2} q^{2} \mathbf{M}(0)+\frac{c q\left[\sigma_{-}, \mathbf{M}(0)\right]}{c^{2}-1}-\frac{c^{2} \sigma_{-} \mathbf{M}(0) \sigma_{-}}{\left(c^{2}-1\right)^{2}} \\
& +\frac{\mathbf{M}(0)}{\left(c^{2}-1\right)^{2}} \int_{0}^{T} \mathbf{F}_{0}(y)^{-1} \sigma_{-} \mathbf{F}_{0}(y) d y
\end{aligned}
$$

where $q:=c T /\left(c^{2}-1\right) \in \mathbb{R}$. (See [9], Section 5 , for details.) With the information at hand, taking the trace in the series and using Abel's identity one can obtain the expansions

$$
\begin{gathered}
\operatorname{det}(\mathbf{M}(\lambda))=1+2 q \lambda+2 q^{2} \lambda^{2}+O\left(\lambda^{3}\right), \\
\operatorname{tr}(\mathbf{M}(\lambda))=2+2 q \lambda+\left(q^{2}+\kappa\right) \lambda^{2}+O\left(\lambda^{3}\right),
\end{gathered}
$$


as $\lambda \rightarrow 0$, where

$$
\kappa:=\frac{M_{12}(0)}{\left(c^{2}-1\right)^{2}} \int_{0}^{T} F_{11}(y, 0)^{2} d y .
$$

3.2. The modulational stability index. Now we extract information about the behavior of the spectrum $\sigma$ in a complex neighborhood of the origin in the complex plane. We also compute expansions of the Floquet multipliers near the origin, and of the function $D(\lambda, \mu)$ in a full complex neighborhood of $(\lambda, \mu)=(0,1)$. (For related constructions in other problems, see $[2,7]$ for the generalized $\mathrm{KdV}$ equation, and [5] for the BBM equation.) The Floquet multipliers are roots of $D(\lambda, \mu)=0$, that is

$$
\mu=\mu_{ \pm}(\lambda)=\frac{1}{2}\left(\operatorname{tr}(\mathbf{M}(\lambda)) \pm\left((\operatorname{tr}(\mathbf{M}(\lambda)))^{2}-4 \operatorname{det}(\mathbf{M}(\lambda))\right)^{1 / 2}\right) .
$$

To analyze the multipliers near $\lambda=0$, we first calculate the quadratic discriminant with the help of the expansions (3.5) and (3.6). We obtain:

$$
(\operatorname{tr}(\mathbf{M}(\lambda)))^{2}-4 \operatorname{det}(\mathbf{M}(\lambda))=4 \kappa \lambda^{2}+O\left(\lambda^{3}\right), \quad \lambda \rightarrow 0 .
$$

Using (3.6), the first few terms in the Taylor series about $\lambda=0$ of the (analytic) Floquet multipliers are:

$$
\mu_{ \pm}(\lambda)=1+\left(q \pm \kappa^{1 / 2}\right) \lambda+O\left(\lambda^{2}\right), \quad \lambda \rightarrow 0 .
$$

Noting that $\kappa$ is proportional to the monodromy matrix element $M_{12}(0)$ by strictly positive factors because $F_{11}(z, 0)$ is a differentiable function satisfying $F_{11}(0,0)=1$, this formula motivates the definition of the "modulational" instability index:

Definition 3.2. The modulational instability index is given by

$$
\gamma_{\mathrm{M}}:=\operatorname{sgn}\left(M_{12}(0)\right)=\operatorname{sgn}\left(-\left(c^{2}-1\right) T_{E}\right),
$$

with the understanding that $\gamma_{\mathrm{M}}:=0$ if $M_{12}(0)=0$ (or equivalently, if $T_{E}=0$ ). In particular, $\gamma_{\mathrm{M}}=1$ for rotational waves of any speed.

Lemma 3.3. The periodic Evans function $D\left(\lambda, e^{i \theta}\right)$ is analytic in the variables $(\lambda, \theta) \in \mathbb{C}^{2}$ and has the following expansion in a neighborhood of $(\lambda, \theta)=(0,0)$ :

$$
D\left(\lambda, e^{i \theta}\right)=-\kappa \lambda^{2}+(i \theta-q \lambda)^{2}+O(3),
$$

where $O(3)$ denotes terms of order three or higher in $(\lambda, \theta)$.

Proof. This follows from the formula $D\left(\lambda, e^{i \theta}\right)=e^{2 i \theta}-\operatorname{tr}(\mathbf{M}(\lambda)) e^{i \theta}+\operatorname{det}(\mathbf{M}(\lambda))$ upon expanding the exponentials in power series about $\theta=0$, substituting the expansions (3.5) and (3.6), and using the definition (3.7).

The index $\gamma_{\mathrm{M}}$ determines the behavior of the spectrum near the origin (see Lemma 6.12 in $[9])$.

Lemma 3.4. If $\gamma_{\mathrm{M}}=1$ but $\kappa \neq q^{2}>0$, then the equation $D\left(\lambda, e^{i \theta}\right)=0$ parametrically describes (for small real $\theta$ ) two smooth curves passing through the origin tangent to the imaginary axis in a neighborhood of the origin the complex $\lambda$-plane. If $\gamma_{\mathrm{M}}=-1$ then the equation $D\left(\lambda, e^{i \theta}\right)=0$ instead parametrically describes two distinct smooth curves that cross at the origin with tangent lines making acute non-zero angles with the imaginary axis.

The presence of any spectrum $\sigma$ that is not purely imaginary implies spectral instability. The particular type of instability detected by the condition $\gamma_{\mathrm{M}}=-1$ is called a strong modulational instability, for which we give the following formal definition. 
Definition 3.5. A periodic traveling wave solution $f$ of the Klein-Gordon equation (1.1) is said to be modulationally unstable (or, to have a modulational instability) if for every neighborhood $U$ of the origin $\lambda=0,(\sigma \backslash i \mathbb{R}) \cap U \neq \emptyset$. Otherwise, $f$ is said to be modulationally stable. The modulational instability is strong if near the origin there is spectrum $\sigma$ in the cone $(\operatorname{Re} \lambda)^{2}>\delta(\operatorname{Im} \lambda)^{2}$ for some $\delta>0$.

3.3. Relation to Whitham's modulation theory. There is an important relationship between the modulational instability index $\gamma_{\mathrm{M}}$ and the ellipticity/hyperbolicity of Whitham's system of equations in formal modulation theory (justifying, in this fashion, its name). Let us define the classical action as

$$
W(E, c):=\left(c^{2}-1\right) \int_{0}^{T} f_{z}(z ; E, c)^{2} d z .
$$

Theorem $3.6([9])$. Suppose $c^{2} W_{E}^{2}+W W_{E E} \neq 0$. Whitham's system of modulation equations (cf. [12]) is hyperbolic (resp., elliptic) if and only if $\gamma_{\mathrm{M}}=1$ (resp., $\gamma_{\mathrm{M}}=-1$ ), where $\gamma_{\mathrm{M}}$ is the modulational instability index.

If $T_{E}=0$, then simultaneously $\gamma_{\mathrm{M}}=0$ and the Whitham system is on the borderline between the hyperbolic and elliptic cases with a double real characteristic velocity. Theorem 3.6 analytically confirms, in the case of the Klein-Gordon equation (1.1), the following well-accepted fact: if the Whitham modulation system is elliptic, then the underlying periodic traveling wave is spectrally unstable. This relationship has been explored in other contexts as well (cf. $[2,6])$.

\section{Stability And instability Results}

4.1. Modulational instability. The first applications of the modulational instability index concern instability results for librational waves.

Theorem 4.1 ([9]). Let $V$ be a potential satisfying the assumptions above. A librational periodic traveling wave solution of the Klein-Gordon equation (1.1) for which $\left(c^{2}-1\right) T_{E}>0$ holds is strongly modulationally unstable.

Since modulational instability implies spectral instability, we have the following:

Corollary $4.2([9])$. All librational waves satisfying $\left(c^{2}-1\right) T_{E}>0$ are spectrally unstable. In particular, all librational traveling wave solutions of the sine-Gordon equation (1.2) are strongly modulationally unstable and hence spectrally unstable.

4.2. (In)stability results for rotational waves. Since $\gamma_{\mathrm{M}}=1$ for rotational waves (see Proposition 1.1), the former are modulationally stable. This is inconclusive, however, for spectral stability. In the generic case of $\kappa \neq q^{2}>0$, the spectrum $\sigma$ is locally tangent to the imaginary axis at the origin $\lambda=0$, but these curves could fail to be confined to the imaginary axis, or because there could be other parts of the spectrum $\sigma$ with nonzero real parts far from the origin. In other words, there could be other instabilities which cannot be detected by $\gamma_{\mathrm{M}}$. To conclude the spectral stability analysis we proved the following result.

Theorem 4.3 ([9]). Under the assumptions on $V$ above we have the following: (i) all periodic traveling waves of the Klein-Gordon equation (1.1) of superluminal rotational type are spectrally unstable; and, (ii) all periodic traveling waves of the Klein-Gordon equation (1.1) of subluminal rotational type are spectrally stable. 
The proofs of statements (i) and (ii) are quite different. The proof of (i) is based on the introduction of a spectrum-detecting function $G: \mathbb{C} \rightarrow \mathbb{R}$ defined in terms of the Floquet multipliers: $G(\lambda):=\log \left|\mu_{+}(\lambda)\right| \log \left|\mu_{-}(\lambda)\right|$. The proof of (ii) involves a direct calculation of $\sigma$ using energy estimates. For details, see Section 7 in [9]. Theorem 4.3 generalizes the spectral stability results for the sine-Gordon equation obtained in [8]:

Corollary 4.4 ([11, 8]). Periodic wavetrains for the sine-Gordon equation (1.2) of librational type, as well as superluminal rotational waves, are spectrally unstable. Subluminal rotational sine-Gordon periodic waves are spectrally stable.

It was Scott [11] who first analyzed the spectral stability of sine-Gordon wavetrains. Although Scott's conclusion turned out to be correct, his proof was not. The results in [8] provided the first completely rigorous proof of Scott's observations, which have been long accepted as fact in the nonlinear wave propagation literature. For extensions of these and other stability results to more complicated potentials, the reader is referred to [9].

\section{REFERENCES}

[1] J. Alexander, R. A. Gardner, and C. K. R. T. Jones, A topological invariant arising in the stability analysis of travelling waves, J. Reine Angew. Math. 410 (1990), pp. 167-212.

[2] J. C. Bronski and M. A. Johnson, The modulational instability for a generalized KdV equation, Arch. Rational Mech. Anal. 197 (2010), no. 2, pp. 357-400.

[3] C. Chicone, The monotonicity of the period function for planar Hamiltonian vector fields, J. Differential Equations 69 (1987), no. 3, pp. 310-321.

[4] R. A. Gardner, On the structure of the spectra of periodic travelling waves, J. Math. Pures Appl. (9) 72 (1993), no. 5, pp. 415-439.

[5] M. A. Johnson, On the stability of periodic solutions of the generalized Benjamin-Bona-Mahony equation, Phys. D 239 (2010), no. 19, pp. 1892-1908.

[6] M. A. Johnson And K. Zumbrun, Rigorous justification of the Whitham modulation equations for the generalized Korteweg-de Vries equation, Stud. Appl. Math. 125 (2010), no. 1, pp. 69-89.

[7] M. A. Johnson, K. Zumbrun, and J. C. Bronski, On the modulation equations and stability of periodic generalized Korteweg-de Vries waves via Bloch decompositions, Phys. D 239 (2010), no. 23-24, pp. 2057-2065.

[8] C. K. R. T. Jones, R. Marangell, P. D. Miller, and R. G. Plaza, On the stability analysis of periodic sine-Gordon traveling waves, Phys. D 251 (2013), no. 1, pp. 63-74.

[9] - Spectral and modulational stability of periodic wavetrains for the nonlinear Klein-Gordon equation, J. Differential Equations 257 (2014), no. 12, pp. 4632-4703.

[10] A. C. Sсотт, A nonlinear Klein-Gordon equation, Am. J. Phys. 37 (1969), no. 1, pp. 52-61.

[11] — Waveform stability on a nonlinear Klein-Gordon equation, Proc. IEEE 57 (1969), no. 7, pp. 1338-1339.

[12] G. B. Whitham, Non-linear dispersive waves, Proc. Roy. Soc. Ser. A 283 (1965), pp. 238-261.

(C. K. R. T. Jones) Department of Mathematics, University of North Carolina, Chapel Hill, NC 27599 (U.S.A.)

E-mail address: ckrtj@amath.unc.edu

(R. Marangell) School of Mathematics and Statistics F07, University of Sydney, Sydney NSW 2006 (Australia)

E-mail address: robert.marangell@sydney.edu.au

(P. D. Miller) Department of Mathematics, University of Michigan, Ann Arbor, Mi 48109 (U.S.A.)

E-mail address: millerpd@umich.edu

(R. G. Plaza) Instituto de Investigaciones en Matemáticas Aplicadas y en Sistemas, Universidad Nacional Autónoma de México, Apdo. Postal 20-726, C.P. 01000 México D.F. (Mexico)

E-mail address: plaza@mym.iimas.unam.mx 